CVIA

EDITORIAL

pISSN 2508-707X / eISSN 2508-7088 https://doi.org/10.22468/cvia.2018.00080 CVIA 2018;2(2):37-37

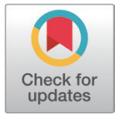

\section{Japanese Special Issue of Cardiovascular Imaging Asia}

\author{
Teruhito Mochizuki \\ Vice-President, Asian Society of Cardiovascular Imaging \\ Department of Radiology, Ehime University School of Medicine, Ehime, Japan
}

Received: March 30, 2018

Accepted: April 5, 2018
I am grateful to the Cardiovascular Imaging Asia (CVIA) editorial office for planning the Japanese Special Issue. Seven Japanese experts in cardiac imaging and its analysis contributed manuscripts to this issue.

When I started cardiac computed tomography (4D-CT, coronary CT angiography) in 1996 using single-slice helical CT, I was excited by its potential, and I was sure that cardiac CT would be clinically useful. By 2004, CANON (formerly TOSHIBA) Medical Systems exhibited a 256-row detector at the annual meeting of the Radiological Society of North America, which was used in 2005 to image patients' hearts and a moving cardiac phantom.

As the development of CT systems, i.e., hardware and reconstruction algorithms, has continued, coronary CT angiography has become a gateway for cardiac imaging of coronary artery disease. Beyond coronary CT angiography, various cardiac CT applications such as CT perfusion, CT fractional flow reserve, myocardial blood flow quantification, CT strain, and 4D functional analysis, among others, are now available. Magnetic resonance (MR) also has considerable potential for cardiac imaging. MR has markedly improved with regard to image quality and acquisition methods, which can shorten acquisition time without loss of image quality. Various clinical applications for cardiac MR are currently being implemented. Nuclear cardiology is not included in this issue; however, semiconductor detector single-photon emission computed tomography with higher sensitivity and spatial resolution is now available. New positron emission tomography myocardial perfusion radiopharmaceuticals such as ${ }^{18} \mathrm{~F}$ Fulupiridaz, which have higher extraction fractions, have also shown promise.

I would like to thank Dr. Kakuya Kitagawa for his effort to arrange this special issue.

I hope this Japan-focused special issue will serve to emphasize the continuing enhancement and expansion of cardiac imaging in Asian counties. 